\title{
A new adaptive control of dual-motor driving servo system with backlash nonlinearity
}

\author{
HAIBO ZHAO ${ }^{1,2, *}$ and CHENGGUANG $\mathrm{WANG}^{3}$ \\ ${ }^{1}$ Engineering Technology Research Center of Optoelectronic Appliance, Tongling 244061, Anhui Province, \\ People's Republic of China \\ ${ }^{2}$ School of Electrical Engineering, Tongling University, Tongling 244061, Anhui Province, People's Republic \\ of China \\ ${ }^{3}$ Sichuan Institute of Aerospace System Engineering, Chengdu 610100, Sichuan Province, People's Republic of China \\ e-mail: happyzhaohaibo@126.com; cgw81421@163.com
}

MS received 26 August 2017; revised 17 January 2018; accepted 26 January 2018; published online 10 August 2018

\begin{abstract}
In order to weaken the influence of backlash nonlinearity on a dual-motor driving servo system, we first establish the state-space model of the system. We then propose a new adaptive controller combining a projection algorithm with backstepping control for the first time, to the best of our knowledge, and analyze its stability. In the simulation analysis, we respectively choose a triangular wave, sawtooth wave, and random signal as the input signal. Simulation results validate a higher tracking accuracy and stronger adaptability of the proposed control law than that of mere backstepping control. In the experimental tests, we respectively choose a step signal and sine signal and simultaneously apply a white noise signal to the system output after $3 \mathrm{~s}$ in each test. The test results validate a stronger adaptability and robustness than that of mere backstepping control.
\end{abstract}

Keywords. Adaptive control; backstepping control; dual-motor driving; projection algorithm; backlash nonlinearity.

\section{Introduction}

In mechanical transmission systems, the existence of backlash nonlinearity will affect the system's dynamic performance and steady-state precision [1-3]. Hence, it is necessary to study the control strategy of weakening the effect of backlash nonlinearity on the system. The commonly used models of backlash nonlinearity include the hysteretic model [4], the dead-zone model [5, 6], and the impact model [7]. In addition, some control strategies, such as the adaptive inverse method [8,9], robust adaptive control [10-12], fuzzy logic control [13, 14], neural network control [15, 16], dynamic surface control [17], and the describing function method [18] have been successfully used by some scholars. However, backlash nonlinearity is still a challenging problem because of its non-differential characteristic.

Because of the effect of backlash nonlinearity, the system not only produces output error, but its stability is also reduced due to the limit cycle oscillation. In addition, the gear's mutual impact also produces serious oscillation and noise. In recent years, many scholars have also studied the control strategies of backlash nonlinearity. In [19], Tarbouriech et al. focused on the stability analysis and the control design for a linear system with a backlash operator

*For correspondence in the input based on Lyapunov techniques. In [20], an adaptive robust controller for a nonlinear system was designed in the case of unknown input backlash, parameter uncertainty, and external disturbances. Vörös [21] dealt with the parameter identification of nonlinear dynamic systems with input saturation and output backlash using three-block cascade models. An iterative least-squaresbased parameter estimation algorithm with internal variables estimations was proposed and illustrated on examples of simulated nonlinear dynamic systems with saturation in the input block and with backlash in the output block. In [22], a robust method was applied to eliminate the influences of truncation errors and bounded errors in the backlash model on system performance. A nonlinear control system considering the uncertainties of the backlash of nonlinear plants was proposed in [23], and a robust nonlinear control system was designed. However, other scholars [19-23] have not used projection algorithms.

The projection algorithm by itself is mature. For the operation of variable-speed wind generators in [24], the adaptive control scheme was based on the affine projection algorithm, which provides faster convergence and less computational complexity than the least-mean-squares algorithm. In [25], to support fast adaptation to the acoustic echo environment, a linear filter was adapted using an affine projection algorithm. The simulation results confirm 
that the proposed algorithm achieves fast and stable convergence under saturation-type microphone distortion, and can achieve better performance than conventional algorithms. In [26], a projection algorithm was used in backstepping controller design, but the backstepping controller was conventional. An optimal variable step-size affine projection algorithm for modified filtered-x active noise control systems was introduced in [27]. The simulation results on the active noise control environments show both fast convergence rate and low steady-state error.

In this paper, we use a dual-motor driving servo system as the research object. We first establish the state-space model of the system, and then propose a new adaptive control strategy combining a projection algorithm with backstepping control for the first time. In the design process of the controller, we introduce the integration of position tracking error so as to ensure that the system tracking error is asymptotically stable and tends to zero better under the condition of an uncertain system model. Simulation analysis and experimental tests all validate the effectiveness of the proposed control strategy compared with mere backstepping control.

The rest of the paper is organized as follows. System modeling is detailed in Section 2 and design of the proposed controller in Section 3. Simulation analysis is presented in Section 4 to show the effectiveness of the proposed strategy. Section 5 details the experimental tests and Section 6 concludes the paper.

\section{System modeling}

The schematic of the dual-motor driving servo system is shown in Figure 1. $O_{0}$ is the driven-subsystem, and $O_{1}$ and $\mathrm{O}_{2}$ are driving-subsystems. The block diagram of the system is shown in Figure 2.

In order to facilitate the study, we assume that the transmission ratio is 1 and the backlash is $2 \alpha$. From the block diagram, we can obtain the dynamic equation of the dual-motor driving servo system as follows:

$$
\left\{\begin{array}{c}
J_{i} \ddot{\theta}_{i}(t)+b_{i} \dot{\theta}_{i}(t)=u(t)-\tau_{i}(t)+(-1)^{i-1} w \\
J_{m} \ddot{\theta}_{m}(t)+b_{m} \dot{\theta}(t)=\sum_{i=1}^{2} \tau_{i}(t)
\end{array}\right.
$$

where $J_{i}$ and $J_{m}$ are the rotational inertias of the driving wheel and driven wheel, respectively; $b_{i}$ and $b_{m}$ are the

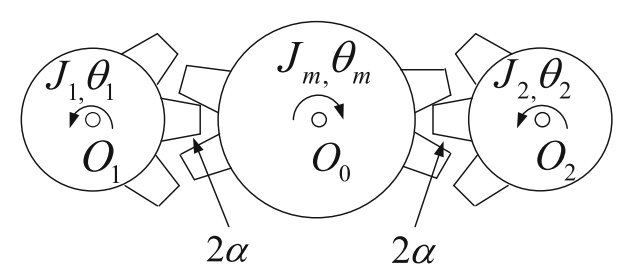

Figure 1. Schematic of dual-motor driving servo system. viscous friction coefficients of the driving wheel and driven wheel, respectively; $\theta_{i}$ and $\theta_{m}$ are the rotational angles of the driving wheel and driven wheel, respectively; $\dot{\theta}_{i}$ and $\dot{\theta}_{m}$ are the rotational speeds of the driving wheel and driven wheel, respectively; $u(t)$ is the input torque of the system; $\tau_{i}(t)$ is the transmitted torque when the driving wheel contacts the driven wheel; and $w$ is the bias torque.

The dead-zone model of backlash can be expressed as follows [28]:

$$
\tau_{i}=\left\{\begin{array}{cc}
k\left(z_{i}(t)+\alpha\right)+c \dot{z}_{i}(t) & z_{i}(t)<-\alpha, \\
0 & \left|z_{i}(t)\right| \leq \alpha \\
k\left(z_{i}(t)+\alpha\right)+c \dot{z}_{i}(t) & z_{i}(t)>\alpha
\end{array}\right.
$$

where $z_{i}(t)=\theta_{i}(t)-\theta_{m}(t)$.

To simplify the controller design, we divide $\tau_{i}$ into three parts:

$$
\tau_{i}=\tau_{i 0}+\Delta \tau_{i a}+\Delta \tau_{i b},
$$

where $\tau_{i 0}$ is the linear transmitted torque, $\Delta \tau_{i a}$ is the nonlinear transmitted torque, $\Delta \tau_{i b}$ is the disturbance of transmitted torque $\tau_{i}$, and their expressions are as follows, respectively:

$$
\tau_{i 0}=k z_{i}(t)
$$

$$
\Delta \tau_{i a}=\left\{\begin{array}{cc}
k \alpha & z_{i}(t)<-\alpha \\
0 & \left|z_{i}(t)\right| \leq \alpha \\
-k \alpha & z_{i}(t)>\alpha
\end{array}\right.
$$

$$
\Delta \tau_{i b}=\left\{\begin{array}{cc}
c \dot{z}_{i}(t) & z_{i}(t)<-\alpha \\
0 & \left|z_{i}(t)\right| \leq \alpha \\
c \dot{z}_{i}(t) & z_{i}(t)>\alpha
\end{array}\right.
$$

In order to simplify the expression, let $\Delta \tau_{i}=\Delta \tau_{i a}+\Delta \tau_{i b}$.

If we choose the state variables of system as $x_{1}=\theta_{m}$, $x_{2}=\dot{\theta}_{m}, x_{3 i}=\theta_{i}, x_{4 i}=\dot{\theta}_{i}$, then the state-space equation model of the system is

$$
\left\{\begin{array}{c}
\dot{x}_{1}=x_{2}, \\
\dot{x}_{2}=\frac{k}{J_{m}}\left(x_{31}+x_{32}\right)-\frac{2 k}{J_{m}} x_{1}-\frac{b_{m}}{J_{m}} x_{2}+\frac{\Delta \tau_{1}+\Delta \tau_{2}}{J_{m}}, \\
\dot{x}_{3 i}=x_{4 i}, \\
\dot{x}_{4 i}=\frac{u}{J_{i}}+\frac{(-1)^{i-1} w}{J_{i}}+\frac{k}{J_{i}} x_{1}-\frac{k}{J_{i}} x_{3 i}-\frac{b_{i}}{J_{i}} x_{4 i}-\frac{\Delta \tau_{i}}{J_{i}}, \\
y=x_{1},
\end{array}\right.
$$

where $y$ is the output of system.

To facilitate the writing of the following expression, we choose $\hbar_{2}=\frac{k}{J_{m}}, \hbar_{21}=\frac{2 k}{J_{m}}, \hbar_{22}=\frac{b_{m}}{J_{m}}, \Delta_{2}=\frac{\Delta \tau_{1}+\Delta \tau_{2}}{J_{m}}, \hbar_{4 i}=\frac{1}{J_{i}}, \hbar_{4 i 1}=$ $\frac{k}{J_{i}}, \hbar_{4 i 3}=\frac{k}{J_{i}}, \hbar_{4 i 4}=\frac{b_{i}}{J_{i}}, \Delta_{4 i}=-\frac{\Delta \tau_{i}}{J_{i}}$.

The simplified state-space equation is expressed as follows: 


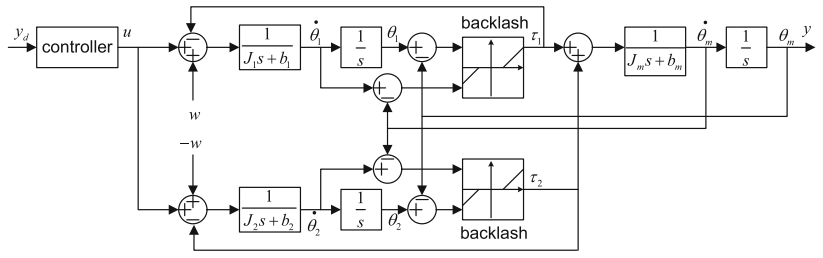

Figure 2. Block diagram of dual-motor driving servo system.

$$
\left\{\begin{array}{l}
\dot{x}_{1}=x_{2}, \\
\dot{x}_{2}=\hbar_{2}\left(x_{31}+x_{32}\right)-\hbar_{21} x_{1}-\hbar_{22} x_{2}+\Delta_{2}, \\
\dot{x}_{3 i}=x_{4 i} \\
\dot{x}_{4 i}=\hbar_{4 i} u+\hbar_{4 i}(-1)^{i-1} w+\hbar_{4 i 1} x_{1}-\hbar_{4 i 3} x_{3 i}-\hbar_{4 i 4} x_{4 i}+\Delta_{4 i}, \\
y=x_{1}
\end{array}\right.
$$

In the actual system, the system parameters $J_{i}, J_{m}, b_{i}, b_{m}$, and $k$ affected by lubrication, temperature, and material wear are uncertain, which causes the change of gear transmitted torque. The design goal of this paper is to design an adaptive controller [29, 30] that makes the system output $y$ asymptotically stable when tracking the expected output $y_{d}$, namely $\lim _{t \rightarrow \infty}\left|y-y_{d}\right|=0$.

\section{Controller design}

We can see that $O_{1}$ and $O_{2}$ have a parallel relationship from Eq. (8), and they are series-connected with $O_{0}$. Moreover, Eq. (1) is a second-order nonlinear uncoupled differential equation of $\theta_{i}$ and $\theta_{m}$.

Assumption 1 The system states $\theta_{m}, \dot{\theta}_{m}, \theta_{i}$, and $\dot{\theta}_{i}$ are all measurable.

Assumption 2 Suppose that the fourth derivative of $y_{d}$ exists.

Assumption 3 System parameters $J_{i}, J_{m}, b_{i}, b_{m}, k$, and $\Delta \tau_{i b}$ are all bounded.

Then,

$$
\begin{aligned}
& 0<J_{i \min } \leq J_{i} \leq J_{i \max }, \\
& 0<J_{m \min } \leq J_{m} \leq J_{m \max }, \\
& 0<b_{i \min } \leq b_{i} \leq b_{i \max }, \\
& 0<b_{m \min } \leq b_{m} \leq b_{m \max }, \\
& 0<k_{\min } \leq k \leq k_{\max } \\
& 0<\left|\Delta_{\tau_{i b}}\right| \leq \Delta \tau_{i b \max }
\end{aligned}
$$

\subsection{Projection algorithm}

A projection algorithm itself is mature, and there are three kinds of projection algorithms commonly used at present: successive projection [31], discontinuous projection [32], and smooth projection [33]. Since we use the backstepping method to design the controller, we must take the derivative of the controller repeatedly, so we use the smooth projection method to ensure the smoothness of the controller.

For parameter estimation vector $\hat{\vartheta}=\left[\hat{\vartheta}_{1}, \ldots, \hat{\vartheta}_{p}\right]$, we define the smooth projection mapping $\operatorname{Pr}$ oj $: R^{P} \rightarrow R^{P}$ as follows [33]:

$$
\operatorname{Pr} o j(\hat{\vartheta})=\left[\operatorname{Pr} o j_{1}\left(\hat{\vartheta}_{1}\right), \ldots, \operatorname{Pr} o j_{p}\left(\hat{\vartheta}_{p}\right)\right]^{T},
$$

where $\operatorname{Pr} o j_{i}\left(\hat{\vartheta}_{i}\right)$ meets both of the following requirements:

1) $\operatorname{Pr} o j_{i}\left(\hat{\vartheta}_{i}\right)=\left(\hat{\vartheta}_{i}\right), \forall \hat{\vartheta}_{i} \in \Omega_{i}=\left\{v: \vartheta_{i \min } \leq v \leq \vartheta_{i \max }\right\}$,

2) $\operatorname{Pr} \operatorname{oj}_{i}\left(\hat{\vartheta}_{i}\right) \in\left\{v: \vartheta_{i \min }-\varepsilon_{\vartheta_{i}} \leq v \leq \vartheta_{i \max }+\varepsilon_{\vartheta_{i}}\right\}, \forall \hat{\vartheta}_{i} \in R$..

According to the actual system requirements, we must ensure that the second derivative of the projection function exists, and we choose the projection function as follows [26]:

$\operatorname{Pr} \operatorname{oj}_{i}\left(\hat{\vartheta}_{i}\right)= \begin{cases}\vartheta_{i \max }+\frac{2 \varepsilon_{\vartheta_{i}} \arctan \left(\left(\hat{\vartheta}_{i}-\vartheta_{i \max }\right) / \varepsilon_{\vartheta_{i}} \times \frac{\pi}{2}\right)}{\pi} & \hat{\vartheta}_{i}>\vartheta_{i \max }, \\ \hat{\vartheta}_{i} & \vartheta_{i \min } \leq \hat{\vartheta}_{i} \leq \vartheta_{i \max }, \\ \vartheta_{i \min }-\frac{2 \varepsilon_{\vartheta_{i}} \arctan \left(\left(\hat{\vartheta}_{i}-\vartheta_{i \max }\right) / \varepsilon_{\vartheta_{i}} \times \frac{\pi}{2}\right)}{\pi} & \hat{\vartheta}_{i}<\vartheta_{i \min } .\end{cases}$

\subsection{Backstepping design}

Step 1 Define the position tracking error $e_{1}$ as

$$
e_{1}=x_{1}-y_{d}
$$

We choose the Lyapunov function $V_{1}$ as follows:

$$
V_{1}=\frac{1}{2} e_{1}^{2}
$$

The derivative of $V_{1}$ is

$$
\dot{V}_{1}=e_{1} \dot{e}_{1}=e_{1}\left(\dot{x}_{1}-\dot{y}_{d}\right)=e_{1}\left(x_{2}-\dot{y}_{d}\right)
$$

Let $x_{2}$ be a virtual control variable, suppose the expectation of $x_{2}$ is $\eta_{2}$, and define the error between $x_{2}$ and $\eta_{2}$ as $e_{2}$ :

$$
e_{2}=x_{2}-\eta_{2}
$$

and if

$$
\eta_{2}=-k_{1} e_{1}+\dot{y}_{d}-k_{0} \int_{0}^{t} e_{1}(\tau) d \tau
$$

then

$$
\dot{V}_{1}=-k_{1} e_{1}^{2}+e_{1} e_{2}-k_{0} e_{1} A,
$$

where $k_{0}>0$ and $k_{1}>0$ are adjustable parameters, and $A$ is the integral of position tracking error, $A=\int_{0}^{t} e_{1}(\tau) d \tau$.

Step 2 Let $x_{31}+x_{32}$ be a virtual control variable. We choose the augmented control Lyapunov function (CLF) $V_{2}$ as follows: 


$$
V_{2}=\frac{1}{2} e_{1}^{2}+\frac{1}{2 \hbar_{2}} e_{2}^{2}+\frac{1}{2} k_{0} A^{2}
$$

Its derivative is

$$
\begin{aligned}
& \dot{V}_{2}=-k_{1} e_{1}^{2}+e_{2} \\
& \quad\left(e_{1}+x_{31}+x_{32}-\frac{\hbar_{21}}{\hbar_{2}} x_{1}-\frac{\hbar_{22}}{\hbar_{2}} x_{2}+\frac{\Delta_{2}}{\hbar_{2}}-\frac{\dot{\eta}_{2}}{\hbar_{2}}\right) .
\end{aligned}
$$

Letting

$$
\vartheta_{2}=\left[\begin{array}{lll}
\frac{\hbar_{21}}{\hbar_{2}} & \frac{\hbar_{22}}{\hbar_{2}} & \frac{1}{\hbar_{2}}
\end{array}\right]^{T}, \varphi_{2}=\left[\begin{array}{lll}
-x_{1} & -x_{2} & -\dot{\eta}_{2}
\end{array}\right]^{T},
$$

Eq. (17) can then be simplified as follows:

$$
\dot{V}_{2}=-k_{1} e_{1}^{2}+e_{2}\left(e_{1}+x_{31}+x_{32}+\vartheta_{2}^{T} \varphi_{2}+\frac{\Delta_{2}}{\hbar_{2}}\right) .
$$

Supposing that the expectation of $x_{31}+x_{32}$ is $\eta_{3}$, we use the adaptive robust control algorithm to design $\eta_{3}$ as follows [26]:

$$
\left\{\begin{array}{c}
\eta_{3}=\eta_{3 a}+\eta_{3 s}, \\
\eta_{3 s}=\eta_{3 s 1}+\eta_{3 s 2}, \\
\eta_{3 s 1}=-e_{1}-k_{2} e_{2}, \\
\eta_{3 a}=-\operatorname{Pr} o j\left(\hat{\vartheta}_{2}\right) \varphi_{2},
\end{array}\right.
$$

where $\hat{\vartheta}_{2}$ is the estimated value of $\vartheta_{2}$ :

$$
\dot{\hat{\vartheta}}_{2}=\Gamma_{2} \varphi_{2} e_{2}
$$

In Eq. (19), $\eta_{3 a}$ is the compensating control of the adaptive model designed according to parameter estimation $\hat{\vartheta}_{2}$, $\eta_{3 s}$ is the robust control variable, $\eta_{3 s 1}$ is the compensation for the Lyapunov function $V_{1}, \eta_{3 s 2}$ is the robust feedback control part, and $k_{2}>0$ is an adjustable parameter. In Eq. (20), $\Gamma_{2}$ is a diagonal matrix that can be replaced by a unit matrix.

Define the error between $x_{31}+x_{32}$ and $\eta_{3}$ as $e_{3}$ :

$$
e_{3}=x_{31}+x_{32}-\eta_{3} \text {. }
$$

Define the error between $\vartheta_{2}$ and the projection of $\hat{\vartheta}_{2}$ as follows:

$$
\tilde{\vartheta}_{\text {proj2 }}=\vartheta_{2}-\operatorname{Pr} o j\left(\hat{\vartheta}_{2}\right)
$$

Suppose that $\eta_{3 s 2}$ satisfies the following two conditions simultaneously:

$$
\left\{\begin{array}{l}
e_{2} \cdot \eta_{3 s 2} \leq 0 \\
e_{2}\left(\eta_{3 s 2}+\tilde{\vartheta}_{p r o j 2}^{T} \varphi_{2}+\frac{\Delta_{2}}{\hbar_{2}}\right) \leq \varepsilon_{2},
\end{array}\right.
$$

where $\varepsilon_{2}$ is a positive number that can be required to take any small value. Substituting Eq. (21) into Eq. (18), we obtain

$$
\dot{V}_{2}=-k_{1} e_{1}^{2}-k_{2} e_{2}^{2}+e_{2}\left(e_{3}+\eta_{3 s 2}+\tilde{\vartheta}_{p r o j 2}^{T} \varphi_{2}+\frac{\Delta_{2}}{\hbar_{2}}\right) .
$$

Step 3 Letting $x_{41}+x_{42}$ be a virtual control variable, we choose the augmented CLF $V_{3}$ as follows:

$$
V_{3}=\frac{1}{2} e_{1}^{2}+\frac{1}{2 \hbar_{2}} e_{2}^{2}+\frac{1}{2} e_{3}^{2} .
$$

Its derivative is

$$
\begin{aligned}
\dot{V}_{3}= & -k_{1} e_{1}^{2}-k_{2} e_{2}^{2}+e_{2}\left(\eta_{3 s 2}+\tilde{\vartheta}_{p r o j 2}^{T} \varphi_{2}+\frac{\Delta_{2}}{\hbar_{2}}\right) \\
& +e_{3}\left(x_{41}+x_{42}-\dot{\eta}_{3}+e_{2}\right) .
\end{aligned}
$$

Supposing that the expectation of $x_{41}+x_{42}$ is $\eta_{4}$, define the error between $x_{41}+x_{42}$ and $\eta_{4}$ as $e_{4}$ :

$$
e_{4}=x_{41}+x_{42}-\eta_{4} .
$$

Substituting Eq. (27) into Eq. (26), we obtain

$$
\begin{aligned}
\dot{V}_{3}= & -k_{1} e_{1}^{2}-k_{2} e_{2}^{2}+e_{2}\left(\eta_{3 s 2}+\tilde{\vartheta}_{p r o j 2}^{T} \varphi_{2}+\frac{\Delta_{2}}{\hbar_{2}}\right) \\
& -k_{3} e_{3}^{2}+e_{3} e_{4},
\end{aligned}
$$

where $k_{3}>0$ is an adjustable parameter.

Step 4 Choose the augmented CLF $V_{4}$ as follows:

$$
V_{4}=\frac{1}{2} e_{1}^{2}+\frac{1}{2 \hbar_{2}} e_{2}^{2}+\frac{1}{2} e_{3}^{2}+\frac{1}{2\left(\hbar_{41}+\hbar_{42}\right)} e_{4}^{2} .
$$

The derivative of $V_{4}$ is expressed as follows:

$$
\begin{aligned}
\dot{V}_{4}= & -k_{1} e_{1}^{2}-k_{2} e_{2}^{2}+e_{2}\left(\eta_{3 s 2}+\tilde{\vartheta}_{\text {proj } 2}^{T} \varphi_{2}+\frac{\Delta \tau_{2}}{\hbar_{2}}\right)-k_{3} e_{3}^{2} \\
& +e_{4}\left(e_{3}+u+\frac{\hbar_{41}-\hbar_{42}}{\hbar_{41}+\hbar_{42}} w+\frac{\hbar_{411}+\hbar_{421}}{\hbar_{41}+\hbar_{42}} x_{1}\right. \\
& -\frac{\hbar_{413}}{\hbar_{41}+\hbar_{42}} x_{31} \cdot-\frac{\hbar_{423}}{\hbar_{41}+\hbar_{42}} x_{32}-\frac{\hbar_{414}}{\hbar_{41}+\hbar_{42}} x_{41} \\
& \left.-\frac{\hbar_{424}}{\hbar_{41}+\hbar_{42}} x_{42}+\frac{\Delta_{41}+\Delta_{42}}{\hbar_{41}+\hbar_{42}}-\frac{1}{\hbar_{41}+\hbar_{42}} \dot{\eta}_{4}\right) .
\end{aligned}
$$

Letting

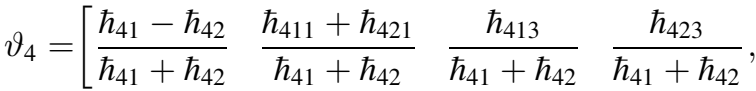

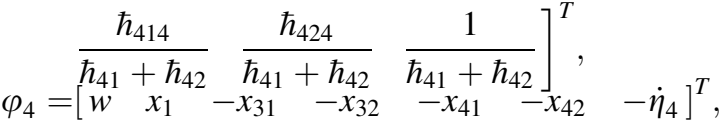

Eq. (30) can then be simplified as follows: 


$$
\begin{aligned}
\dot{V}_{4}= & -k_{1} e_{1}^{2}-k_{2} e_{2}^{2}+e_{2}\left(\eta_{3 s 2}+\tilde{\vartheta}_{\text {proj } 2}^{T} \varphi_{2}+\frac{\Delta \tau_{2}}{\hbar_{2}}\right)-k_{3} e_{3}^{2} \\
& +e_{4}\left(e_{3}+u+\vartheta_{4}^{T} \varphi_{4}+\frac{\Delta_{41}+\Delta_{42}}{\hbar_{41}+\hbar_{42}}\right) .
\end{aligned}
$$

Next, we use the adaptive robust control algorithm to design $u$ as follows:

$$
\left\{\begin{array}{c}
u=u_{a}+u_{s}, \\
u_{s}=u_{s 1}+u_{s 2}, \\
u_{s 1}=-k_{4} e_{4}-e_{3}, \\
u_{a}=-\operatorname{Pr} \operatorname{oj}\left(\hat{\vartheta}_{4}\right) \varphi_{4},
\end{array}\right.
$$

where $\hat{\vartheta}_{4}$ is the estimated value of $\vartheta_{4}$ :

$$
\dot{\hat{\vartheta}}_{4}=\Gamma_{4} \varphi_{4} e_{4} .
$$

In Eq. (32), $u_{a}$ is the compensating control of the adaptive model designed according to the parameter estimation $\vartheta_{4}$. $u_{s}$ is the robust control variable, $u_{s 1}$ is the compensation for the Lyapunov function $V_{3}, u_{s 2}$ is the robust feedback control part, and $k_{4}>0$ is an adjustable parameter. In Eq. (33), $\gamma_{4}$ is a diagonal matrix that can be replaced by a unit matrix. Define the error between $\vartheta_{4}$ and the projection of $\hat{\vartheta}_{4}$ as follows:

$$
\tilde{\vartheta}_{p r o j 4}=\vartheta_{4}-\operatorname{Pr} \operatorname{oj}\left(\hat{\vartheta}_{4}\right) .
$$

Suppose that $u_{s 2}$ satisfies the following two conditions simultaneously:

$$
\left\{\begin{array}{c}
e_{4} \cdot u_{s 2} \leq 0, \\
e_{4}\left(u_{s 2}+\tilde{\vartheta}_{p r o j 4}^{T} \varphi_{4}+\frac{\Delta_{41}+\Delta_{42}}{\hbar_{41}+\hbar_{42}}\right) \leq \varepsilon_{4},
\end{array}\right.
$$

where $\varepsilon_{4}$ is a positive number that can be required to take any small value. Substituting Eq. (32) into Eq. (31), we obtain

$$
\begin{aligned}
\dot{V}_{4}= & -k_{1} e_{1}^{2}-k_{2} e_{2}^{2}+e_{2}\left(\eta_{3 s 2}+\tilde{\vartheta}_{\text {proj } 2}^{T} \varphi_{2}+\frac{\Delta \tau_{2}}{\hbar_{2}}\right)-k_{3} e_{3}^{2} \\
& +e_{4}\left(u_{s 2}+\tilde{\vartheta}_{\text {proj } 4}^{T} \varphi_{4}+\frac{\Delta_{41}+\Delta_{42}}{\hbar_{41}+\hbar_{42}}\right)-k_{4} e_{4}^{2} .
\end{aligned}
$$

\subsection{Stability analysis}

Theorem 1 The error variable $e=\left(e_{1}, e_{2}, e_{3}, e_{4}\right)$ of the closed-loop system containing controlled object (8), control law (32), and adaptive law (33) is bounded.
Simultaneously, e converges to a sufficiently small neighborhood of the origin according to the exponential law.

Proof From Eqs. (23), (35), and (36), we can obtain

$$
\begin{aligned}
\dot{V}_{4} & \leq-k_{1} e_{1}^{2}-k_{2} e_{2}^{2}-k_{3} e_{3}^{2}-k_{4} e_{4}^{2}+\varepsilon_{2}+\varepsilon_{4} \\
& \leq-d_{0} V_{4}+\varepsilon_{0},
\end{aligned}
$$

where

$$
\begin{aligned}
d_{0} & =\min \left(2 k_{1}, 2 \hbar_{2} k_{2}, 2 k_{3}, 2\left(\hbar_{41}+\hbar_{42}\right) / k_{4}\right), \\
\varepsilon_{0} & =\varepsilon_{2}+\varepsilon_{4} .
\end{aligned}
$$

Calculating the integral on both sides of Eq. (37), we obtain:

$$
V_{4}(t) \leq e^{-d_{0} t} V_{4}(0)+\frac{\varepsilon_{0}}{d_{0}}\left(1-e^{-d_{0} t}\right),
$$

According to Eqs. (29) and (38), we have

$$
\begin{gathered}
e_{1}^{2} \leq 2 V_{4}(t), \lim _{t \rightarrow \infty} e_{1}(t) \leq \sqrt{\frac{2 \varepsilon_{0}}{d_{0}}} \\
e_{2}^{2} \leq 2 \hbar_{2} V_{4}(t), \lim _{t \rightarrow \infty} e_{2}(t) \leq \sqrt{\frac{2 \hbar_{2} \varepsilon_{0}}{d_{0}}} \\
e_{3}^{2} \leq 2 V_{4}(t), \lim _{t \rightarrow \infty} e_{3}(t) \leq \sqrt{\frac{2 \varepsilon_{0}}{d_{0}}} \\
e_{4}^{2} \leq 2\left(\hbar_{41}+\hbar_{42}\right) V_{4}(t), \lim _{t \rightarrow \infty} e_{4}(t) \leq \sqrt{\frac{2\left(\hbar_{41}+\hbar_{42}\right) \varepsilon_{0}}{d_{0}}} .
\end{gathered}
$$

Thus, the error variable $e=\left(e_{1}, e_{2}, e_{3}, e_{4}\right)$ is bounded. In addition, in the condition of adjustable parameters $k_{1}, k_{2}$, $k_{3}, k_{4}$ have been chosen, we can adjust $\varepsilon_{2}$ and $\varepsilon_{4}$ to ensure that $\varepsilon_{0}$ is small sufficiently, which can ensure that the error variable $e=\left(e_{1}, e_{2}, e_{3}, e_{4}\right)$ converges to a sufficient small neighborhood of the origin according to the exponential law.

\section{Simulation analysis}

The system physical parameters are as follows:

$$
\begin{aligned}
J_{1} & =J_{2}=0.185 \mathrm{~kg} \cdot \mathrm{m}^{2}, b_{1}=b_{2}=1.2 \mathrm{~N} \cdot \mathrm{m} \cdot \mathrm{s} / \mathrm{rad}, \\
J_{m} & =0.028 \mathrm{~kg} \cdot \mathrm{m}^{2}, \alpha=0.5 \mathrm{rad}, b_{m}=1.3 \mathrm{~N} \cdot \mathrm{m} \cdot \mathrm{s} / \mathrm{rad}, \\
k & =[560+2 \sin (\pi t)] \mathrm{N} \cdot \mathrm{m} / \mathrm{rad}, w=50 \mathrm{~N} \cdot \mathrm{m}, \\
c & =[0.15+0.01 \sin (\pi t)] \mathrm{N} \cdot \mathrm{m} / \mathrm{rad}, b_{1}=b_{2} \\
& =1.2 \mathrm{~N} \cdot \mathrm{m} \cdot \mathrm{s} / \mathrm{rad} .
\end{aligned}
$$

The system initial conditions are as follows: 


$$
x_{1}=0, x_{2}=0, x_{3 i}=0, x_{4 i}=0, u(0)=0 .
$$

The initial estimates of the system parameters are chosen as follows:

$$
\begin{aligned}
& \hat{\vartheta}_{2}(0)=\left[\begin{array}{lll}
8.2 & 0.0064 & 0.000749
\end{array}\right]^{T}, \\
& \hat{\vartheta}_{4}(0)=\left[\begin{array}{lllllll}
-0.039 & 486 & 52.65 & 55.76 & 0.42 & 0.48 & 0.034
\end{array}\right]^{T} \text {. }
\end{aligned}
$$

The system simulation is completed in the MATLAB6.5 (MathWorks, USA) environment, and we choose the design parameters as follows:

$$
\begin{aligned}
& k_{1}=32, k_{2}=12, k_{3}=9, k_{4}=4, \varepsilon_{2}=0.08, \varepsilon_{4}=25, \\
& \Gamma_{2}=\operatorname{diag}[1,1,1], \Gamma_{4}=\operatorname{diag}[1,1,1,1] .
\end{aligned}
$$

Figures 3 and 4 show the response curves of the system tracking triangular wave using mere backstepping control (MBC) and combination control of a projection algorithm and backstepping control (PABC), respectively. Figures 5 and 6 show the error curves of the system tracking triangular wave using MBC and PABC, respectively. Figures 7 and 8 show the response curves of the system tracking sawtooth wave using $\mathrm{MBC}$ and PABC, respectively. Figures 9 and 10 show the error curves of the system tracking sawtooth wave using $\mathrm{MBC}$ and $\mathrm{PABC}$, respectively. Figures 11 and 12 show the response curves of the system tracking random signal using $\mathrm{MBC}$ and $\mathrm{PABC}$, respectively. Figures 13 and 14 show the error curves of the system tracking random signal using $\mathrm{MBC}$ and $\mathrm{PABC}$, respectively. From Figures 3-10, we can see that PABC has not only better dynamic tracking performance, but also smaller tracking error than MBC. From Figures 11-14, it is obvious that PABC not only has smaller tracking error, but also stronger adaptability than MBC.

\section{Experimental tests}

The block diagram of the dual-motor driving servo system is shown in Figure 15 and the experimental system is shown in Figure 16 [17]. The following graphics are the comparison charts of $\mathrm{MBC}$ and PABC.

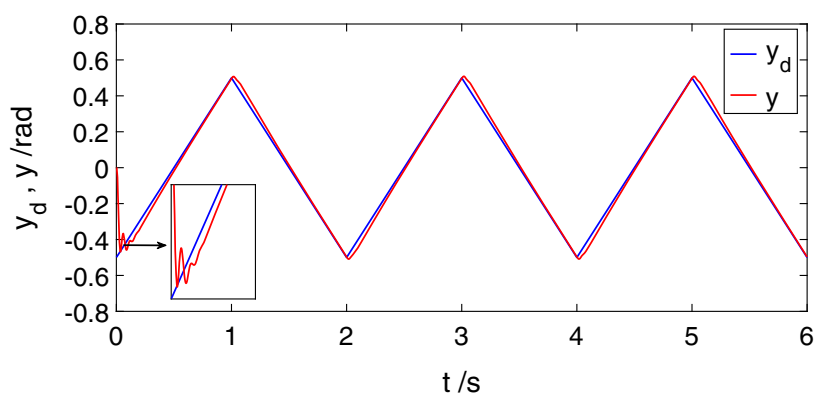

Figure 3. Tracking triangular wave using MBC.

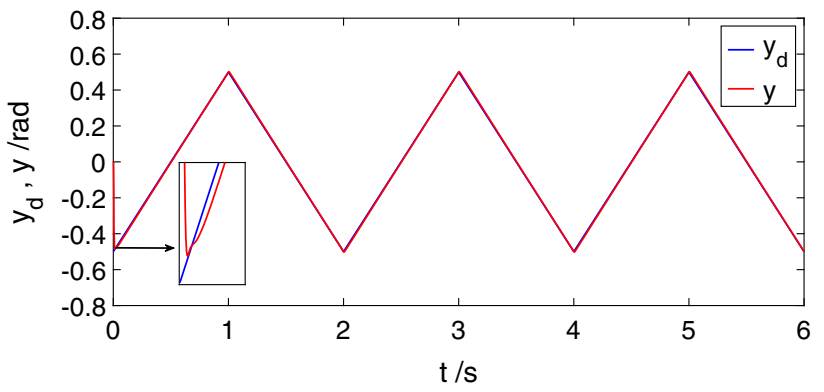

Figure 4. Tracking triangular wave using PABC.

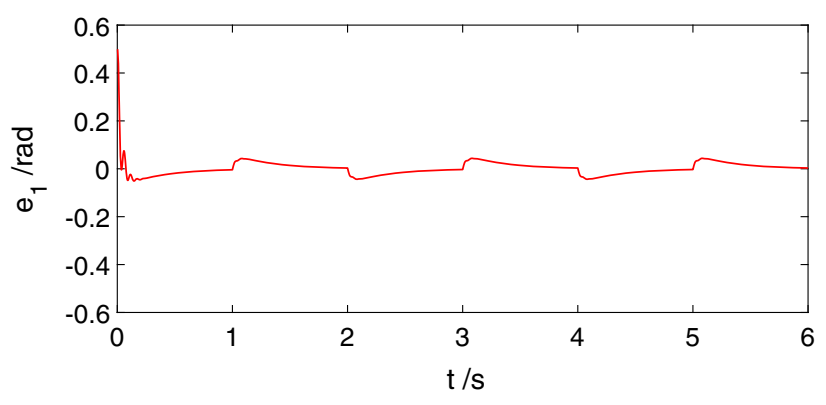

Figure 5. Error of tracking triangular wave using MBC.

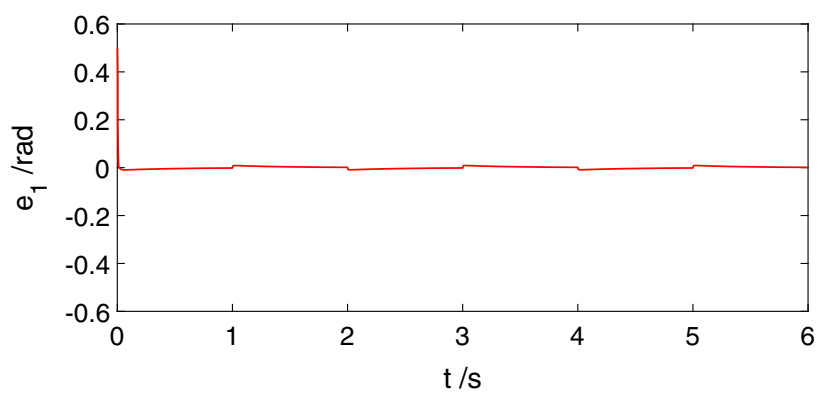

Figure 6. Error of tracking triangular wave using PABC.

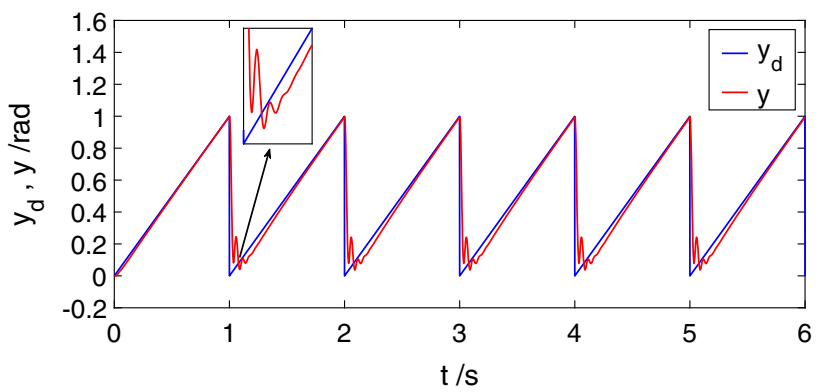

Figure 7. Tracking sawtooth wave using MBC. 


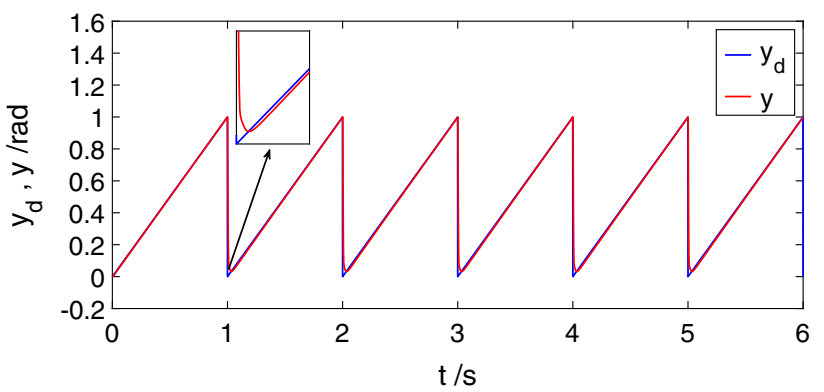

Figure 8. Tracking sawtooth wave using PABC.

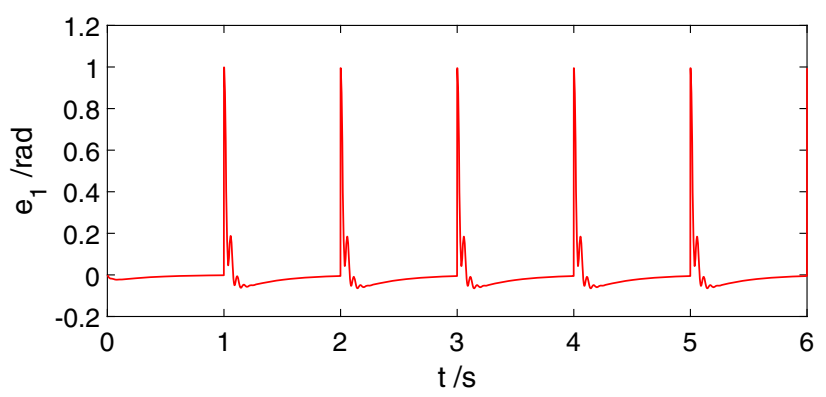

Figure 9. Error of tracking sawtooth wave using MBC.

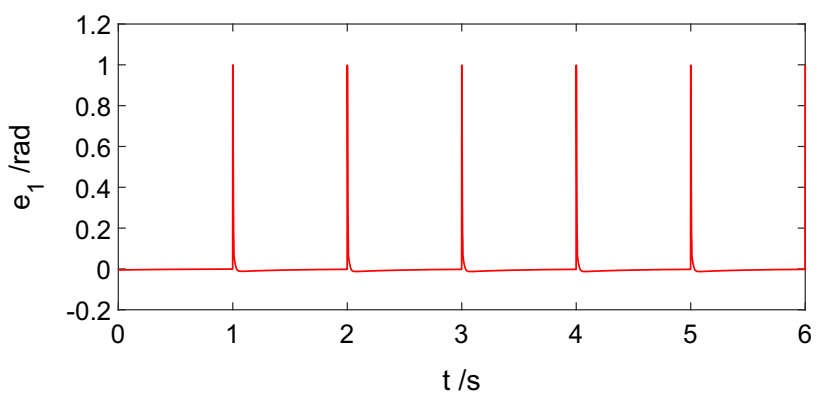

Figure 10. Error of tracking sawtooth wave using PABC.

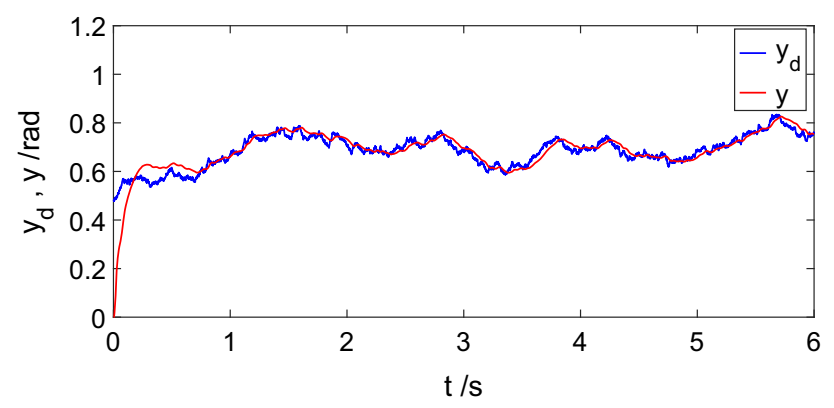

Figure 11. Tracking random signal using MBC.

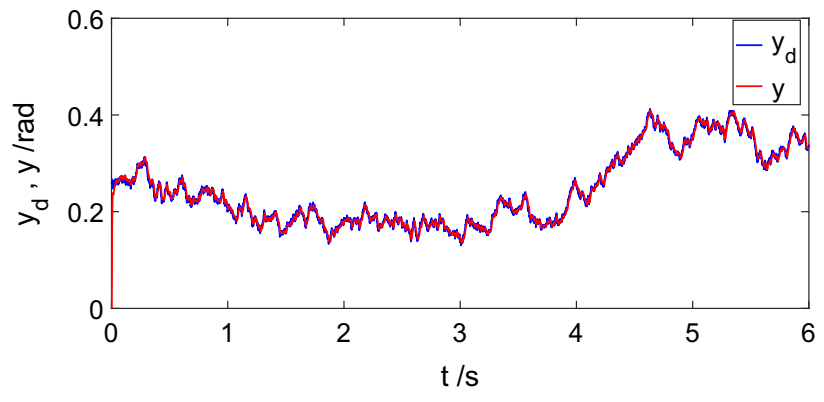

Figure 12. Tracking random signal using $P A B C$.

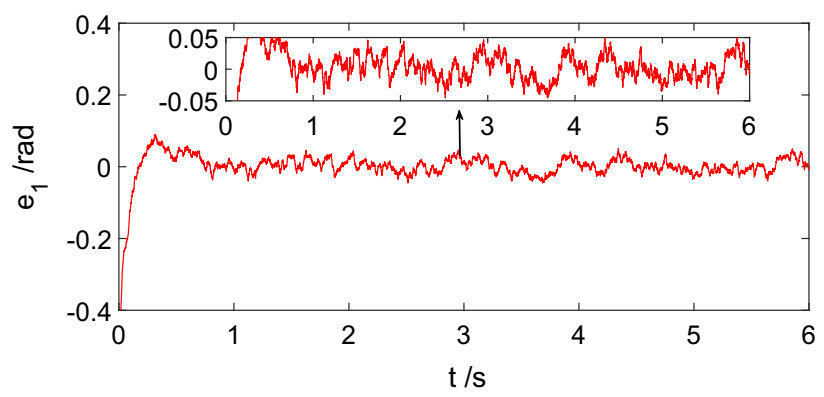

Figure 13. Error of tracking random signal using MBC.

\subsection{System hardware implementation}

The dual-motor driving servo system consists of a computer control system, inverter, AC motor, gear drive chain, and digital signal processing (DSP) unit.

1) Computer control system. The computer control system completes signal sampling, system monitoring, and control quantity acquisition, and mainly includes a data sampling board, electric anti-backlash board, shaft encoder board, etc.

In this system, the PC/104 computer uses a SCM/SPT4 module (SBS Science \& Technology Co., Ltd., China). The data sampling data uses a SysExpanModule/ADT700 type (SBS Science \& Technology Co., Ltd. ).

The electric anti-backlash board is custom built by us, and its main function is to generate the bias torque and speed-error negative feedback signal. At the same time, in order to solve the problem of system electromagnetic compatibility, the interface board also has a photoelectric isolation circuit. The board has a certain generality and can be applied to other systems.

The shaft encoder board uses an AD2S80AUD chip (AD Corp., USA) as well as a shaft encoder to convert the position of the system output axis to 16-bit natural binary code; the shaft encoder then sends it to the PC/104 computer to realize position feedback. 


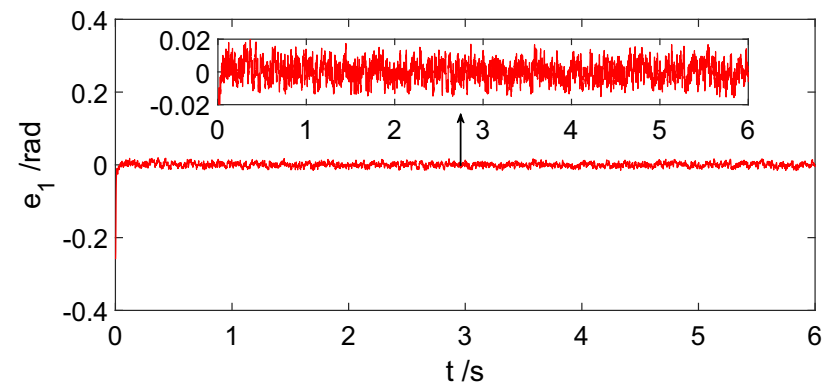

Figure 14. Error of tracking random signal using PABC.

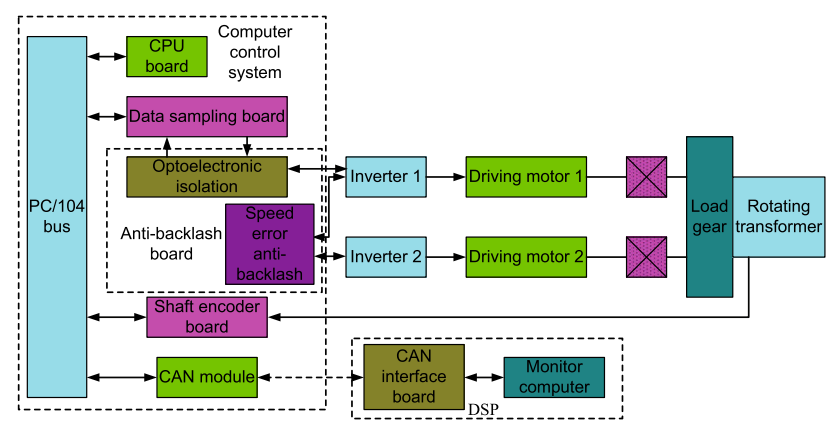

Figure 15. Block diagram of dual-motor driving servo system.

2) Inverter and $A C$ motor. The Inverter uses a $C D / S$ series CR06250 driver (Kollmorgen, Inc., USA). The motor is a Kollmorgen M-405-C type.

3) Gear drive chain. The rotating transformer and the load gear are coaxially connected, and they are combined with the shaft encoder board to form the angular position feedback module. A 110XFSW008 rotating transformer was used.

4) DSP unit. A TMS320F2812 32-bit fixed-point DSP chip (Texas Instruments Corp., USA) was used for digital signal processing. The TMS320LF2812 chip formed a controller area network (CAN) interface card and CAN intelligent node, and the eCAN module of the TMS320LF2812 chip was used to connect the CAN bus driver, so that the node can be connected arbitrarily to any network with a CAN bus.

\subsection{System software implementation}

The system software framework is shown in Figure 17, mainly composed of a main program, interrupt handling routine, and algorithm subroutine. The interrupt handling routine contains a timer interrupt, serial port interrupt, CAN receive interrupt, and power protect interrupt. The algorithm subroutine contains a control algorithm and angular displacement planning. All the program code is

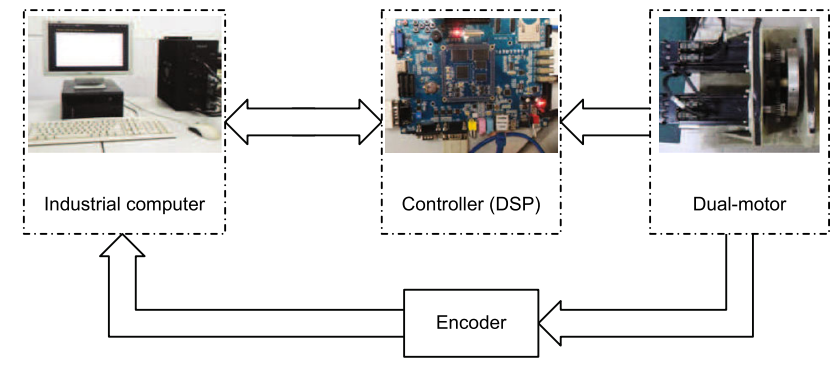

Figure 16. Experimental system.

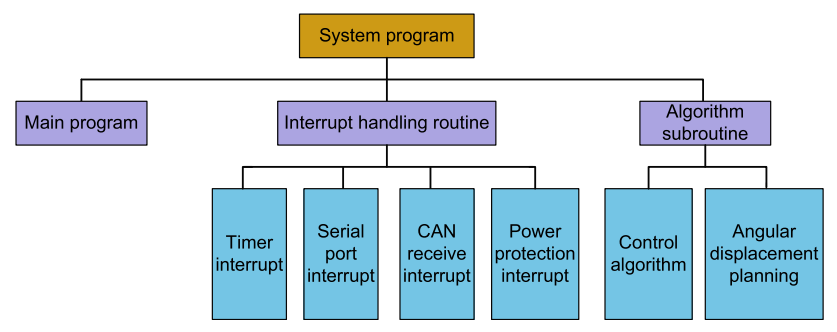

Figure 17. System software framework.

implemented in the $\mathrm{C}$ language in the DSP development environment CCS3.3; an output file is then generated, which was burned to 2812 Flash for debugging.

\subsection{System experiment}

Figures 18 and 19 show the system response curves to a step signal using MBC and PABC, respectively. Figures 20 and 21 show the system response curves to a sine signal $y_{d}=\sin (0.4 \pi t)$ using $\mathrm{MBC}$ and $\mathrm{PABC}$, respectively. Simultaneously, we applied a white noise signal to the system output after $3 \mathrm{~s}$ in each experimental test. From Figures 18 and 19, we can see that the system response has larger overshoot and larger tracking error using MBC. However, the system response has almost no overshoot when using $\mathrm{PABC}$, and the tracking error is also smaller than that of MBC. After $3 \mathrm{~s}$, it is obvious that the system

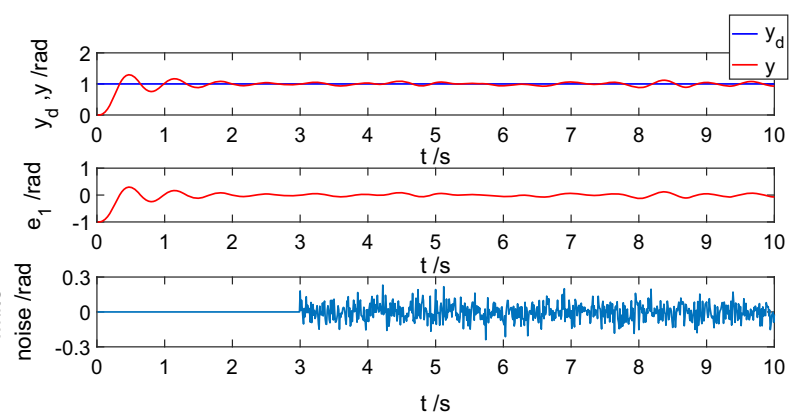

Figure 18. System response to step signal using MBC. 

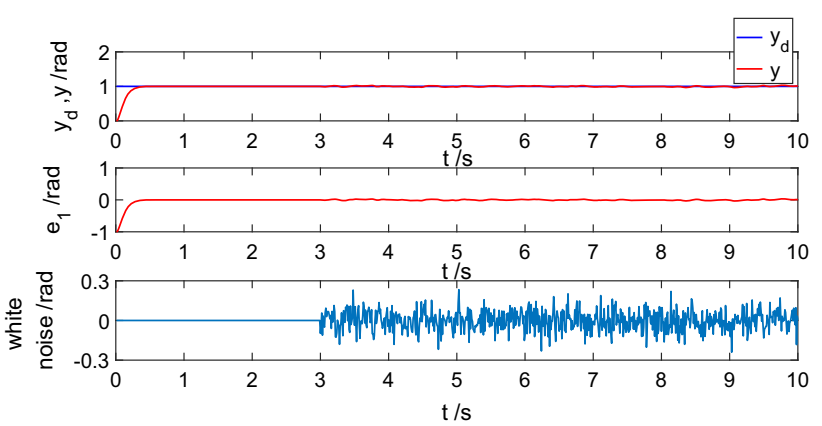

Figure 19. System response to step signal using PABC.
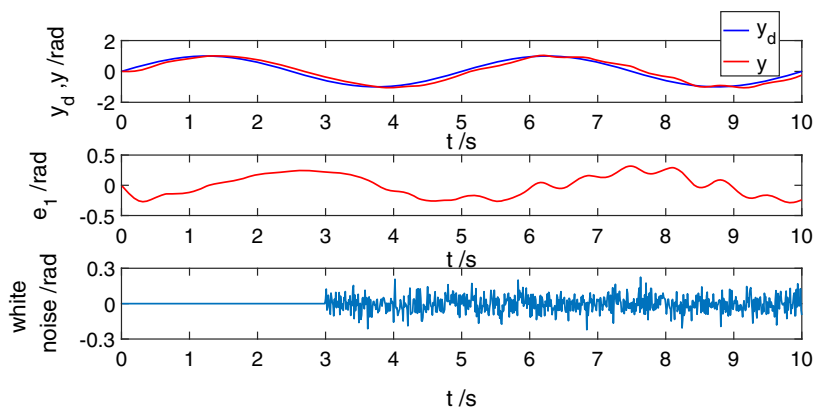

Figure 20. System response to sine signal using MBC.

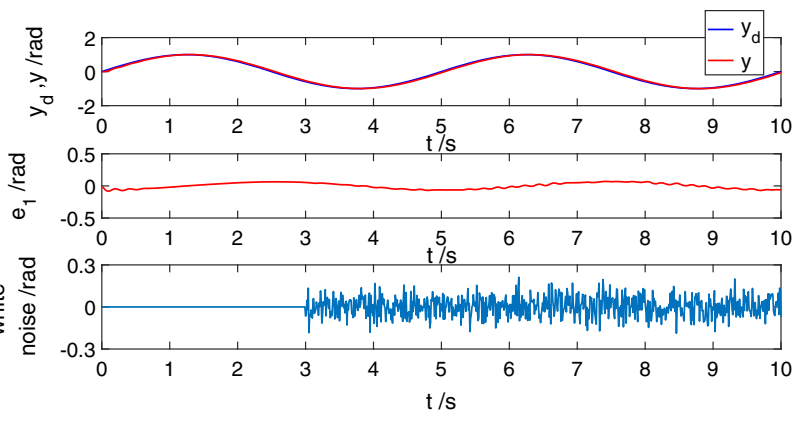

Figure 21. System response to sine signal using PABC.

response fluctuation amplitude of $\mathrm{PABC}$ is obviously smaller than that of MBC. From Figures 20 and 21, we can see that the tracking error and response fluctuation amplitude after $3 \mathrm{~s}$ of PABC are also smaller than those of MBC.

From the experimental tests, we can see that PABC has smaller tracking error and higher anti-interference ability than those of MBC.

\section{Conclusions}

Aimed at solving the problem of the parameter uncertainty of dual-motor driving servo systems, we proposed, for the first time, to the best of our knowledge, a new adaptive control algorithm combining a projection algorithm with backstepping control. Simulation results show that the system can not only track the desired output asymptotically stably by using the proposed control algorithm, but that it also has higher tracking accuracy and stronger adaptability than that of mere backstepping control. Experimental test results show that the proposed control algorithm has better adaptability and stronger robustness than those of mere backstepping control, validating the effectiveness of the proposed control algorithm. However, even though friction nonlinearity also exists in dual-motor driving servo systems, we did not consider the coexistence of friction and backlash nonlinearity here, but have deferred it to planned future work.

\section{Acknowledgements}

This work was supported by the National Natural Science Foundation of China (Grant No. 61074023), the Natural Science Foundation in Anhui Province of China (Grant No. 1508085MF130), the Natural Science Research Key Project of Universities in Anhui Province of China (Grant No. KJ2015A297), the Engineering Technology Research Center of Optoelectronic Appliance of Anhui Province, China, and the Sichuan Institute of Aerospace System Engineering of Sichuan Province, China.

\section{References}

[1] Ma Y L, Huang J and Zhang D 2009 Adaptive compensation of backlash nonlinearity for servo systems. J. Syst. Simul. 21: 1498-1504

[2] Moradi H and Salarieh H 2012 Analysis of nonlinear oscillations in spur gear pairs with approximated modelling of backlash nonlinearity. Mech. Mach. Theory. 51: 14-31

[3] Shi Z G and Zuo Z Y 2015 Backstepping control for gear transmission servo systems with backlash nonlinearity. IEEE Trans. Autom. Sci. Eng. 12: 752-757

[4] Mousavi S H, Ranjbar-Sahraei B and Noroozi N 2012 Output feedback controller for hysteretic time-delayed MIMO nonlinear systems: an H-based indirect adaptive interval type-2 fuzzy approach. Nonlinear Dyn. 68: 63-76

[5] Liu W J, Shyu K K and Hsu K C 2011 Design of uncertain multi-input systems with state delay and input deadzone nonlinearity via sliding mode control. Int. J. Control Autom. Syst. 9: 461-469

[6] Du RH, Wu Y F, Chen W and Chen Q W 2013 Adaptive backstepping fuzzy control for servo systems with backlash. Control Theory Appl. 30: 254-260

[7] Yang S, Fafitis A and Wiezel A 2012 Nonlinear impact model of a tennis racket and a ball. J. Mech. Sci. Technol. 26: $315-321$

[8] Tao G and Kokotovic P V 1993 Adaptive control of systems with backlash. Automatica 29: 323-335 
[9] Tao G and Kokotovic P V 1995 Continuous-time adaptive control of systems with unknown backlash. IEEE Trans. Automat. Control 40: 1083-1087

[10] Su CY et al 2000 Robust adaptive control of a class of nonlinear systems with unknown backlash-like hysteresis. IEEE Trans. Automat. Control 45: 2427-2432

[11] Zhou J, Zhang C and Wen C 2007 Robust adaptive output control of uncertain nonlinear plants with unknown backlash nonlinearity. IEEE Trans. Automat. Control 52: 503-509

[12] Guo J, Yao B, Wu Y F and Chen Q W 2010 Adaptive robust control for a class of nonlinear uncertain system with input backlash. Control Decis. 25: 1580-1584

[13] Jang J O and Jeon G J 2006 Backlash compensation of nonlinear systems using fuzzy logic. Int. J. Syst. Sci. 37: 485-492

[14] Cazarez-Castro N R et al 2010 Fuzzy logic control with genetic membership function parameters optimization for the output regulation of a servomechanism with nonlinear backlash. Expert Syst. Appl. 37: 4368-4378

[15] Selmic R R and Lewis F L 2000 Neural net backlash compensation with Hebbian tuning using dynamic inversion. Automatica 37: 1269-1277

[16] Zhang DX et al 2008 Neural network sliding mode control approach to backlash and friction compensation. J. Univ. Electr. Sci. Technol. Chin. 37: 793-796

[17] Sun G F 2014 Dynamic surface control for nonlinear systems and the application to servo systems. Doctor's degree thesis, Beijing Institute of Technology. Beijing City of China

[18] Zhao H B and Zhu Y G 2013 A compensation approach of dead-zone nonlinearity in dual-motor driving servo system. Appl. Mech. Mater. 389: 454-459

[19] Tarbouriech S et al 2014 Stability analysis and stabilization of systems with input backlash. IEEE Trans. Automat. Contr. 59: 488-494

[20] Guo J et al 2011 Adaptive control of a nonlinear system with input backlash. Acta Armamentarii 32: 1298-1304

[21] Vörös J 2014 Identification of nonlinear dynamic systems with input saturation and output backlash using three-block cascade models. J. Franklin Inst. 351: 5455-5466
[22] Zhu S et al 2012 Adaptive control of a class of periodically time-varying nonlinear systems with input backlash. Control Theory Appl. 29: 535-538

[23] Hanawa T and Deng M C 2014 Compensation on the uncertain nonlinear plants preceded by uncertain backlash. IEEJ Trans. Electron. Inf. Syst. 134: 1214-1220

[24] Hasanien H M and Muyeen S M 2015 Affine projection algorithm based adaptive control scheme for operation of variable-speed wind generator. IET Gener. Transm. Dis. 9: 2611-2616

[25] Lee $\mathrm{K}$ et al 2015 Nonlinear acoustic echo cancellation using a nonlinear postprocessor with a linearly constrained affine projection algorithm. IEEE Trans. Circuits Syst. II Express Briefs. 62: 881-885

[26] Yuan X X 2014 The research of the modeling and control of servo system with large inertia based on multidrive to eliminate backlash. Master's degree thesis, Nanjing University of Science and Technology. Nanjing City of China

[27] Song J M and Park P G 2015 An optimal variable step-size affine projection algorithm for the modified filtered-x active noise control. Signal Process. 114: 100-111

[28] Zhao H B and Zhou X H 2010 Dual-motor driving servo system based on fuzzy RBF neural network. J. Anhui Univ. Technol. Sci. 25(1): 57-61

[29] Yadav A K and Gaur P 2014 AI-based adaptive control and design of autopilot system for nonlinear UAV. Sadhana 39: 765-783

[30] Hariprasad K and Bhartiya S 2014 Adaptive robust model predictive control of nonlinear systems using tubes based on interval inclusions. In: Proceedings of the IEEE Conference on Decision and Control. Los Angeles, CA, USA, pp. 2032-2037

[31] Wang J J et al 2016 Successive projections algorithm-based three-band vegetation index for foliar phosphorus estimation. Ecol. Indic. 67: 12-20

[32] Busquets E et al 2015 Discontinuous projection-based adaptiverobust control for displacement-controlled actuators. J. Dyn. Syst. Meas. Control Trans. ASME 137(8): 081007

[33] Teel AR 1993 Adaptive tracking with robust stability. In: Proceedings of the IEEE Conference on Decision and Control San Antonio, TX, USA, Dec. pp. 570-575 